\title{
A High-order Internal Model Based Iterative Learning Control Scheme for Discrete Linear Time-varying Systems
}

\author{
Wei Zhou ${ }^{1,2}$ Miao $\mathrm{Yu}^{1} \quad$ De-Qing Huang ${ }^{3}$ \\ ${ }^{1}$ College of Electrical Engineering, Zhejiang University, Hangzhou 310027, China \\ ${ }^{2}$ College of Engineering, Jiangsu Institute of Commerce, Nanjing 211100, China \\ ${ }^{3}$ Department of Aeronautics, Imperial College London, London SW7 2AZ, UK
}

\begin{abstract}
In this paper, an iterative learning control algorithm is proposed for discrete linear time-varying systems to track iterationvarying desired trajectories. A high-order internal model (HOIM) is utilized to describe the variation of desired trajectories in the iteration domain. In the sequel, the HOIM is incorporated into the design of learning gains. The learning convergence in the iteration axis can be guaranteed with rigorous proof. The simulation results with permanent magnet linear motors (PMLM) demonstrate that the proposed HOIM based approach yields good performance and achieves perfect tracking.
\end{abstract}

Keywords: Iterative learning control, high-order internal model, discrete linear time-varying systems, iteration-varying desired trajectory, permanent magnet linear motors.

\section{Introduction}

The idea of iterative learning control (ILC) was generated firstly by Uchiyama in $1978^{[1]}$. The basic objective of ILC is to overcome the imperfect knowledge of the plant using previous tracking information and to achieve output tracking through repetition ${ }^{[2-5]}$. This makes ILC schemes particularly useful in applications with repetitive tasks, such as robot manipulator ${ }^{[6]}$, tracking problem of the taking-off and landing planes ${ }^{[7]}$, freeway traffic control ${ }^{[8]}$, waste-water treatment ${ }^{[9]}$, piezoelectric positioning stage system ${ }^{[10]}$, heat flux boundary control ${ }^{[11]}$, etc. The ILC algorithm has been developed widely from classical ILC $^{[12]}$, higher-order $\mathrm{ILC}^{[13,14]}$, robust $\operatorname{ILC}^{[15,16]}$, optimal $\operatorname{ILC}^{[17]}$ to adaptive ILC $^{[18-22]}$ for the last two decades.

In the conventional ILC, the desired trajectories are assumed to be invariant from iteration to iteration. However, the tracking tasks can be different in different iterations sometimes. For instance, a robot arm is scheduled to move objects tracking one trajectory in the time interval $[0, T]$, then it may move objects tracking another trajectory in the next working process. And another example of a robot manipulator moving an object of mass $m$ in the odd cycles and another object of mass $2 m$ in the even cycle, as was explained in [23]. All these iteration varying problems are called non-repetitiveness in general. Thus, it is helpful that ILC could be designed to harness the non-repetitiveness. Considering the non-repetitiveness

\footnotetext{
Regular Paper

Special Issue on Latest Advances in ILC/RLC Theory and Applications

Manuscript received April 27, 2014; accepted October 28, 2014

This work was supported by National Basic Research Program of China (973 Program)(No. 2012CB316400) and National Natural Science Foundation of China (Nos. 61171034 and 61273134).

Recommended by Guest Editor Rong-Hu Chi

(C) Institute of Automation, Chinese Academy of Science and Springer-Verlag Berlin Heidelberg 2015
}

of the dynamic system, it can be divided into two circumstances: known variation pattern and unknown variation pattern of non-repetitiveness. To reflect known variation pattern of non-repetitiveness, an effective approach is to incorporate a high-order internal model (HOIM) to describe the non-repetitiveness in iteration domain. For instance, the iteration-varying desired trajectories in a robot manipulator can be formulated by an HOIM. In [24], a survey on HOIM applications of different ILC problems was given. According to the internal model principle ${ }^{[25]}$, the generator of known variation pattern of non-repetitiveness must be involved in the ILC controller to improve tracking performance. To reflect iteration varying property of reference trajectories, a high-order internal model (HOIM) was introduced to describe the variations along the iteration axis in [26]. An HOIM-based ILC has been studied recently for iteration-varying reference trajectories for continuous-time linear time-varying systems, and both the initial resetting condition and extension to nonlinear cases are also explored ${ }^{[27]}$. And for continuous-time nonlinear systems, an HOIM-based P-type ILC scheme was presented for tracking iteration-varying trajectories ${ }^{[28]}$. In [27, 28], time-weighted norm method was used to prove the learning convergence in the iteration axis. Moreover, for nonlinear system with time-iteration-varying parameters, an HOIMbased ILC was proposed and composite energy function was used to derive convergence properties of the method ${ }^{[29]}$.

In real implementation, it is necessary to discretize the system dynamics. Researchers have devoted a lot of efforts on ILC for discrete-time systems. A 2-D system theory based ILC has been studied for discrete linear timeinvariant systems ${ }^{[30]}$. A unified learning scheme was considered for the initial shift problem of nonlinear systems with well-defined relative degree ${ }^{[31]}$. In [32], a feed-forward ILC method was designed and updated by past control data 
in the previous trials for a class of discrete-time nonlinear time-varying systems with initial state error, input disturbance and output measurement noise. Another ILC design was proposed to overcome the uncertainties and disturbances for discrete time nonlinear dynamic system ${ }^{[33]}$. And an adaptive ILC scheme was presented to deal with timevarying parametric uncertainties for discrete-time system and can achieve point-wise convergence over a finite interval under random initial states and iteration-varying reference trajectory ${ }^{[34]}$. Yu et al. ${ }^{[35]}$ presented ILC for discrete-time systems with unknown control directions under the framework of adaptive ILC. An adaptive iterative learning control method combining with an $n$-step ahead predictor was employed for a class of nonlinear output feedback discretetime systems with random initial conditions and iterationvarying desired trajectories ${ }^{[36]}$.

As a summary of the above discussions, ILC for discretetime systems tracking iteration-varying desired trajectories is definitely an important issue in practical applications. In this work, an HOIM-based P-type ILC law is proposed for a class of discrete linear time-varying systems with the relative degree of one, and a sufficient condition is derived under which the convergence of the learning process is guaranteed. The effectiveness of the proposed ILC algorithm is illustrated by means of simulation results with permanent magnet linear motors (PMLM).

\section{Problem formulation}

Consider a discrete linear time-varying system with the relative degree of one as

$$
\left\{\begin{array}{l}
x_{k}(t+1)=A(t) x_{k}(t)+B(t) u_{k}(t) \\
y_{k}(t)=C(t) x_{k}(t)
\end{array}\right.
$$

where the subscripts $k$ and $t$ denote the iteration number and discrete time index, matrix $A(t) \in \mathbf{R}^{n \times n}, B(t) \in$ $\mathbf{R}^{n \times m}, C(t) \in \mathbf{R}^{m \times n}$ are uniformly bounded for all $t \in$ $[0, T]$, state $x_{k}(t) \in \mathbf{R}^{n}, u_{k}(t)$ and $y_{k}(t)$ are input and output vectors of the $k$-th iteration, $u_{k}(t) \in \mathbf{R}^{m}, y_{k}(t) \in \mathbf{R}^{m}$, $C(t+1) B(t)$ is full column rank, and the system is minimum phased.

In this work, we consider the iteration-varying reference trajectories $y_{k+1}^{r}(t)$ which are related to the reference trajectories of the past iterations. The variations of desired trajectories along the iteration axis can be expressed by a high-order internal model as

$$
y_{k+1}^{r}(t)=h_{1} y_{k}^{r}(t)+h_{2} y_{k-1}^{r}(t)+\cdots+h_{m} y_{k-(m-1)}^{r}(t)
$$

where $h_{i}, i=1,2, \cdots, m$, are coefficients of a polynomial

$$
S(z)=z^{m}-h_{1} z^{m-1}-h_{2} z^{m-2}-\cdots-h_{m} .
$$

From (2), we can see that the trajectory of the $(k+1)$-th iteration is related to the trajectories of the past $m$ iterations and the HOIM is essentially an auto-regression model in the iteration domain. Then, we consider a shift operator $\omega^{-1[37]}$ which was introduced with the property that $\omega^{-1} y_{k+1}^{r}(t)=y_{k}^{r}(t), \forall t \in[0, T]$. Equality (2) can be rewritten as

$$
y_{k+1}^{r}(t)=H\left(\omega^{-1}\right) y_{k}^{r}(t)
$$

where polynomial $H\left(\omega^{-1}\right)=h_{1}+\cdots+h_{m} \omega^{-m+1}$ is used to describe the high-order internal model.

Definition 1. The $\lambda$-norm ${ }^{[38]}$ is defined for function $f(t)$ as $\|f(t)\|_{\lambda}=\sup _{t \in[0, T]} \mathrm{e}^{-\lambda t}\|f(t)\|$. And the $\lambda$-norm for function $H\left(\omega^{-1}\right) f_{k}(t)$ is defined as

$$
\begin{aligned}
& \left\|H\left(\omega^{-1}\right) f_{k}(t)\right\|_{\lambda}= \\
& \quad\left|h_{1}\right|\left\|f_{k}(t)\right\|_{\lambda}+\cdots+\left|h_{m}\right|\left\|f_{k-m+1}(t)\right\|_{\lambda} .
\end{aligned}
$$

Let $e_{k+1}(t)=y_{k+1}^{r}(t)-y_{k+1}(t)$ be the tracking error at the time instant $t \in[0, T]$ of the $(k+1)$-th iteration. The control objective is to find a control input sequence $u_{k+1}(t)$ for plant (1) such that the output errors $e_{k+1}(t)$ converge to zero as $k \rightarrow \infty$. For the known variation pattern of desired trajectories, the ILC law must embed the characteristic of variation according to internal model principle. To achieve this aim, a P-type ILC which employs the $m$-th order internal model (4) is given as

$$
\begin{aligned}
u_{k+1}(t)= & h_{1} u_{k}(t)+h_{2} u_{k-1}(t)+\cdots+h_{m} u_{k-(m-1)}(t)+ \\
& \gamma_{1} e_{k}(t+1)+\gamma_{2} e_{k-1}(t+1)+\cdots+ \\
& \gamma_{m} e_{k-(m-1)}(t+1)= \\
& H\left(\omega^{-1}\right) u_{k}(t)+\Gamma\left(\omega^{-1}\right) e_{k}(t+1)
\end{aligned}
$$

where $\Gamma\left(\omega^{-1}\right)=\gamma_{1}+\gamma_{2} \omega^{-1}+\cdots+\gamma_{m} \omega^{-m+1}$ and $\gamma_{j}$ is the learning gain. From (6), we can see that to harness the non-repetitiveness in the desired trajectories, the ILC law has to be high-order in the iteration direction.

With respect to the system dynamics (1) and desired trajectories (4), we have the following assumptions:

Assumption 1. Initialization is satisfied throughout repeated trainings, i.e.,

$$
x_{k+1}(0)=H\left(\omega^{-1}\right) x_{k}(0) .
$$

Assumption 2. The matrix $Q(t)$ is defined with bound $b_{Q}=\sup _{t \in[0, T]} Q(t)(Q \in\{A, B, C\})$.

Assumption 3. The polynomial $S(z)$ is stable or critically stable which means that all roots of $S(z)=0$ are within the unit circle or at least one root is lying on the unit circle.

Remark 1. If all the roots of $S(z)=0$ are within the unit circle, the polynomial is stable and the desired trajectories expressed by HOIM will converge to zero as the iteration number approaches infinity. If at least one root is lying on the unit circle, the polynomial is stable and the desired trajectories will vary periodically and never converge to zero. For example, there are two characteristic roots of the polynomial $S_{1}(z)=z^{2}-2 \cos (0.1) z+1$ lying on the unit circle. The desired trajectories generated by $S_{1}(z)$ are $y_{k+1}^{r}(t)=2 \cos (0.1) y_{k}^{r}(t)-y_{k-1}^{r}(t), k=2,3, \cdots$. According to $z$-transform, we have $y_{k}^{r}(t)=A_{1}(t) \cos (0.1 k)+$ $A_{2}(t) \sin (0.1 k)$, where $A_{1}(t)$ and $A_{2}(t)$ are time-varying 
coefficients which are iteration independent and only determined by initial values. It can be seen clearly that the second-order internal model constructed by $S_{1}(z)$ will change periodically and never converge to zero in iteration domain.

\section{Learning convergence analysis}

In this section, we will discuss the convergence property of the proposed HOIM-based ILC.

Theorem 1. For the discrete linear time-varying system (1), given the HOIM-based desired trajectories (4), we consider that the Assumptions 1-3 are satisfied. If the learning gain $\gamma_{j}$ is chosen such that the asymptotic stability of the following polynomial is guaranteed

$$
P(z)=z^{m}-\zeta_{t, k} z^{m-1}-\cdots-\zeta_{t, k-m+1}
$$

where $\zeta_{t, j}=\left\|h_{k+1-j}-C(t+1) B(t) \gamma_{k+1-j}\right\|, t \in[0, T]$, $j \in[k, \cdots, k-m+1]$, then the output error $e_{k}(t)$ converges to zero in $[0, T]$ as $k \rightarrow \infty$ under the HOIM-based ILC law (6), i.e., $\lim _{k \rightarrow \infty} e_{k}(t)=0$.

Proof. First substituting desired trajectories (4) to the $(k+1)$-th tracking error $e_{k+1}(t+1)$, we obtain

$$
\begin{aligned}
e_{k+1}(t+1)= & H\left(\omega^{-1}\right) y_{k}^{r}(t+1)-y_{k+1}(t+1)- \\
& H\left(\omega^{-1}\right) y_{k}(t+1)+H\left(\omega^{-1}\right) y_{k}(t+1)= \\
& H\left(\omega^{-1}\right) e_{k}(t+1)-y_{k+1}(t+1)+ \\
& H\left(\omega^{-1}\right) y_{k}(t+1) .
\end{aligned}
$$

Considering the system dynamics (1), we get

$$
y_{k+1}(t+1)=C(t+1) x_{k+1}(t+1)
$$

and

$$
y_{k}(t+1)=C(t+1) x_{k}(t+1) .
$$

Then, substituting (10) and (11) to (9) yields

$$
\begin{aligned}
& e_{k+1}(t+1)=H\left(\omega^{-1}\right) e_{k}(t+1)+ \\
& \quad H\left(\omega^{-1}\right) C(t+1) x_{k}(t+1)-C(t+1) x_{k+1}(t+1) .
\end{aligned}
$$

From the HOIM-based ILC law (6), we get

$$
\begin{aligned}
& e_{k+1}(t+1)=H\left(\omega^{-1}\right) e_{k}(t+1)- \\
& \quad C(t+1) B(t) \Gamma\left(\omega^{-1}\right) e_{k}(t+1)-C(t+1) A(t) \times \\
& {\left[x_{k+1}(t)-H\left(\omega^{-1}\right) x_{k}(t)\right] .}
\end{aligned}
$$

Taking the norms of (13) and considering Assumption 2, it can be derived that

$$
\begin{gathered}
\left\|e_{k+1}(t+1)\right\| \leq\left\|H\left(\omega^{-1}\right)-C(t+1) B(t) \Gamma\left(\omega^{-1}\right)\right\| \times \\
\left\|e_{k}(t+1)\right\|+b_{C} b_{A}\left\|x_{k+1}(t)-H\left(\omega^{-1}\right) x_{k}(t)\right\| . \quad(14)
\end{gathered}
$$

To evaluate $x_{k+1}(t)-H\left(\omega^{-1}\right) x_{k}(t)$, from plant (1), we have

$$
\begin{gathered}
x_{k+1}(t+1)-H\left(\omega^{-1}\right) x_{k}(t+1)= \\
A(t) x_{k+1}(t)+B(t) u_{k+1}(t)- \\
H\left(\omega^{-1}\right)\left[A(t) x_{k}(t)+B(t) u_{k}(t)\right] .
\end{gathered}
$$

Taking the norms of (15) yields

$$
\begin{gathered}
\left\|x_{k+1}(t+1)-H\left(\omega^{-1}\right) x_{k}(t+1)\right\| \leq \\
\left\|A(t)\left[x_{k+1}(t)-H\left(\omega^{-1}\right) x_{k}(t)\right]\right\|+ \\
\left\|B(t)\left[u_{k+1}(t)-H\left(\omega^{-1}\right) u_{k}(t)\right]\right\| .
\end{gathered}
$$

Applying Assumption 2 and substituting (6) into (16), we have

$$
\begin{gathered}
\left\|x_{k+1}(t+1)-H\left(\omega^{-1}\right) x_{k}(t+1)\right\| \leq \\
b_{A}\left\|x_{k+1}(t)-H\left(\omega^{-1}\right) x_{k}(t)\right\|+ \\
b_{B}\left\|\Gamma\left(\omega^{-1}\right) e_{k}(t+1)\right\| .
\end{gathered}
$$

When $t=0$, the above inequality can be rewritten as

$$
\begin{aligned}
& \left\|x_{k+1}(1)-H\left(\omega^{-1}\right) x_{k}(1)\right\| \leq \\
& b_{A}\left\|x_{k+1}(0)-H\left(\omega^{-1}\right) x_{k}(0)\right\|+ \\
& b_{B}\left\|\Gamma\left(\omega^{-1}\right) e_{k}(1)\right\| .
\end{aligned}
$$

Taking Assumption 1 into account, we have

$$
\left\|x_{k+1}(1)-H\left(\omega^{-1}\right) x_{k}(1)\right\| \leqslant b_{B}\left\|\Gamma\left(\omega^{-1}\right) e_{k}(1)\right\| .
$$

Similarly, when $t=1$, we get

$$
\begin{aligned}
& \left\|x_{k+1}(2)-H\left(\omega^{-1}\right) x_{k}(2)\right\| \leq \\
& b_{A}\left\|x_{k+1}(1)-H\left(\omega^{-1}\right) x_{k}(1)\right\|+ \\
& b_{B}\left\|\Gamma\left(\omega^{-1}\right) e_{k}(2)\right\| .
\end{aligned}
$$

Substituting (19) into (20), it can be obtained that

$$
\begin{aligned}
& \left\|x_{k+1}(2)-H\left(\omega^{-1}\right) x_{k}(2)\right\| \leq \\
& \quad b_{A} b_{B}\left\|\Gamma\left(\omega^{-1}\right) e_{k}(1)\right\|+b_{B}\left\|\Gamma\left(\omega^{-1}\right) e_{k}(2)\right\| .
\end{aligned}
$$

Following the same procedure, we can now conclude that for $t \in[0, T]$,

$$
\begin{aligned}
& \left\|x_{k+1}(t)-H\left(\omega^{-1}\right) x_{k}(t)\right\| \leq \\
& \quad \sum_{j=0}^{t-1} b_{A}^{t-1-j} b_{B}\left\|\Gamma\left(\omega^{-1}\right) e_{k}(j+1)\right\| .
\end{aligned}
$$

Substituting (22) into (14), we have

$$
\begin{aligned}
& \left\|e_{k+1}(t+1)\right\| \leq \\
& \left\|H\left(\omega^{-1}\right)-C(t+1) B(t) \Gamma\left(\omega^{-1}\right)\right\|\left\|e_{k}(t+1)\right\|+ \\
& b_{C} \sum_{j=0}^{t-1} b_{A}^{t-j} b_{B}\left\|\Gamma\left(\omega^{-1}\right) e_{k}(j+1)\right\| .
\end{aligned}
$$

Multiplying both sides of (23) by $\mathrm{e}^{-\lambda(t+1)}$, we can derive from the definition of $\lambda$-norm that

$$
\begin{aligned}
& \sup _{t \in[0, T]} \mathrm{e}^{-\lambda(t+1)}\left\|e_{k+1}(t+1)\right\| \leq \\
& \left\|H\left(\omega^{-1}\right)-C(t+1) B(t) \Gamma\left(\omega^{-1}\right)\right\|\left\|e_{k}\right\|_{\lambda}+ \\
& \sup _{t \in[0, T]} \mathrm{e}^{-\lambda(t+1)} b_{B} b_{C} \sum_{j=0}^{t-1} b_{A}^{t-j}\left\|\Gamma\left(\omega^{-1}\right) e_{k}(j+1)\right\| .
\end{aligned}
$$


Since

$$
\begin{aligned}
& \sup _{t \in[0, T]} \mathrm{e}^{-\lambda(t+1)} b_{B} b_{C} \sum_{j=0}^{t-1} b_{A}^{t-j}\left\|\Gamma\left(\omega^{-1}\right) e_{k}(j+1)\right\| \leq \\
& a^{2}\left\|\Gamma\left(\omega^{-1}\right) e_{k}\right\|_{\lambda} \sum_{j=0}^{t-1} a^{t(1-\lambda)} a^{(\lambda-1) j} \leq \\
& a^{2} \frac{1-a^{T(1-\lambda)}}{a^{(\lambda-1)}-1}\left\|\Gamma\left(\omega^{-1}\right) e_{k}\right\|_{\lambda}
\end{aligned}
$$

where $a=\max \left\{e, b_{A}, b_{B}, b_{C}\right\}$, by expressing the HOIM in the above inequality, we can derive

$$
\begin{aligned}
& a^{2} \frac{1-a^{T(1-\lambda)}}{a^{(\lambda-1)}-1}\left\|\Gamma\left(\omega^{-1}\right) e_{k}\right\|_{\lambda}= \\
& \quad a^{2} \delta\left|\gamma_{1}\right|\left\|e_{k}\right\|_{\lambda}+\cdots+a^{2} \delta\left|\gamma_{m}\right|\left\|e_{k-m+1}\right\|_{\lambda}
\end{aligned}
$$

where $\delta=\frac{1-a^{T(1-\lambda)}}{a^{(\lambda-1)}-1}$. Then, we have

$$
\begin{gathered}
\left\|H\left(\omega^{-1}\right)-C(t+1) B(t) \Gamma\left(\omega^{-1}\right)\right\|\left\|e_{k}\right\|_{\lambda}= \\
\left\|h_{1}-C(t+1) B(t) \gamma_{1}\right\|\left\|e_{k}\right\|_{\lambda}+\cdots+ \\
\left\|h_{m}-C(t+1) B(t) \gamma_{m}\right\|\left\|e_{k-m+1}\right\|_{\lambda} .
\end{gathered}
$$

Substituting (26) and (27) into (24), we can obtain that

$$
\begin{aligned}
& \left\|e_{k+1}\right\|_{\lambda} \leq\left\|h_{1}-C(t+1) B(t) \gamma_{1}\right\|\left\|e_{k}\right\|_{\lambda}+ \\
& a^{2} \delta\left|\gamma_{1}\right|\left\|e_{k}\right\|_{\lambda}+\cdots+ \\
& \left\|h_{m}-C(t+1) B(t) \gamma_{m}\right\|\left\|e_{k-m+1}\right\|_{\lambda}+ \\
& a^{2} \delta\left|\gamma_{m}\right|\left\|e_{k-m+1}\right\|_{\lambda} .
\end{aligned}
$$

Now, we can write the above inequalities from $t=0$ to $t=T$. When $t=0$, we have

$\left\|e_{k+1}(1)\right\|_{\lambda} \leqslant \rho_{0, k}\left\|e_{k}(1)\right\|_{\lambda}+\cdots+\rho_{0, k-m+1}\left\|e_{k-m+1}(1)\right\|_{\lambda}$.

And when $t=T$, we get

$$
\begin{array}{r}
\left\|e_{k+1}(T+1)\right\|_{\lambda} \leq \rho_{T, k}\left\|e_{k}(T+1)\right\|_{\lambda}+\cdots+ \\
\rho_{T, k-m+1}\left\|e_{k-m+1}(T+1)\right\|_{\lambda}
\end{array}
$$

where $\rho_{i, j}=\left\|h_{k+1-j}-C(i+1) B(i) \gamma_{k+1-j}\right\|+$ $a^{2} \delta\left|\gamma_{k+1-j}\right|, i \in[0,1, \cdots, T], j \in[k, k-1, \cdots, k-m+1]$. Thus, we can deduce that

$$
\begin{gathered}
{\left[\begin{array}{c}
\left\|e_{k+1}(1)\right\|_{\lambda} \\
\ldots \\
\left\|e_{k+1}(T+1)\right\|_{\lambda}
\end{array}\right] \leq F_{k}\left[\begin{array}{c}
\left\|e_{k}(1)\right\|_{\lambda} \\
\ldots \\
\left\|e_{k}(T+1)\right\|_{\lambda}
\end{array}\right]+\cdots+} \\
F_{k-m+1}\left[\begin{array}{c}
\left\|e_{k-m+1}(1)\right\|_{\lambda} \\
\cdots \\
\left\|e_{k-m+1}(T+1)\right\|_{\lambda}
\end{array}\right] \\
\text { where } F_{j}=\left[\begin{array}{cccc}
\rho_{0, j} & 0 & \cdots & 0 \\
0 & \rho_{1, j} & \cdots & 0 \\
\cdots & \cdots & & \cdots \\
0 & 0 & \cdots & \rho_{T, j}
\end{array}\right]
\end{gathered}
$$

From (31), we can see that the convergence of $\left\|e_{k+1}\right\|_{\lambda}$ is determined by $F_{s}, s \in[k, k-1, \cdots, k-m+1]$. Consider $\delta$ in (26), we can see that $\delta$ is arbitrarily small with sufficiently large $\lambda$. Thus, we can obtain that the convergence of $\left\|e_{k+1}\right\|_{\lambda}$ is determined by $P(z)=z^{m}-\zeta_{t, k} z^{m-1}-\cdots-$ $\zeta_{t, k-m+1}$, where $\zeta_{t, j}=\left\|h_{k+1-j}-C(t+1) B(t) \gamma_{k+1-j}\right\|$, $t \in[0, T], j \in[k, \cdots, k-m+1]$. If all eigenvalues of $P(z)$ are inside the unit circle, we have $\lim _{k \rightarrow \infty}\left\|e_{k}\right\|_{\lambda}=$ 0 , and from the definition of $\lambda$-norm, we get that $\sup _{t \in[0, T]}\left\|e_{k}(t)\right\| \rightarrow 0$ as $k \rightarrow \infty$. Hence, the convergence of $e_{k}(t) \rightarrow 0$ is obtained as $k \rightarrow \infty$.

\section{Simulation example}

Consider a direct current motor control problem for velocity tracking. The dynamics of a permanent magnet linear motor (PMLM $)^{[39,40]}$ can be described by

$$
\left\{\begin{array}{l}
\dot{x}(t)=v(t) \\
u(t)=k_{1} \psi_{f} \dot{x}(t)+R i(t)+L \dot{i}(t) \\
f_{l}(t)=m \dot{v}(t)+f_{\text {fri }}(t)+f_{\text {rip }}(t)+f_{\text {loa }}(t)+f_{w}(t)
\end{array}\right.
$$

where $x(t)$ and $f_{l}(t)$ are the motor position and the developed force, $v(t)$ is rotor velocity, $u(t), i(t), R$ and $L$ are the voltage, current, resistance and inductance of stator, $k_{1}=\frac{\pi}{\tau}, \tau$ is pole pitch, $\psi_{f}$ is the flux linkage, $m$ is the rotor mass, $f_{\text {fri }}(t), f_{\text {rip }}(t)$ and $f_{\text {loa }}(t)$ denote the frictional, ripple and applied load forces respectively, the term $f_{w}(t)$ includes other uncertainties and disturbances.

Using DQ decomposition theory, we transform the PMLM model (32) by neglecting all the uncertainties and nonlinearities $f_{w}(t)$. We assume that $f_{f r i}(t)+f_{\text {rip }}(t)+$ $f_{\text {loa }}(t)=0$ and $i_{d} \equiv 0$ to simplify the system. Considering $f_{l}=k_{2} \psi_{f} i_{q}(t)$, where $k_{2}=1.5 \frac{\pi}{\tau}$, we have

$$
\left\{\begin{array}{l}
u_{q}(t)=k_{1} \psi_{f} \dot{x}(t)+R i_{q}(t)+L_{q} \dot{i}_{q}(t) \\
k_{2} \psi_{f} i_{q}(t)=m \ddot{x}(t) .
\end{array}\right.
$$

Since $\dot{i}_{q}(t)=\frac{m \dddot{x}(t)}{\left(k_{2} \psi_{f}\right)}$, substituting it into (33), we get

$$
u_{q}(t)=\frac{m \dddot{x}(t)}{k_{2} \psi_{f}}+\frac{R m}{k_{2} \psi_{f}} \ddot{x}(t)+k_{1} \psi_{f} \dot{x}(t) .
$$

Neglecting the third order differential part of (34), we obtain

$$
u_{q}(t)=\frac{R m}{k_{2} \psi_{f}} \ddot{x}(t)+k_{1} \psi_{f} \dot{x}(t) .
$$

Considering $i_{d} \equiv 0$ and replacing $u_{q}$ by $u$, we have

$$
\left\{\begin{array}{l}
\dot{x}(t)=v(t) \\
\dot{v}(t)=-\frac{k_{1} k_{2} \psi_{f}^{2}}{R m} v(t)+\frac{k_{2} \psi_{f}}{R m} u(t) \\
y(t)=v(t)
\end{array}\right.
$$

With the discrete time interval $\Delta=10 \mathrm{~ms}$, the operation cycle is $N=\{0,1, \cdots, 100\}$. Discretizing the system by the 
Euler method yields

$$
\left\{\begin{array}{l}
x(t+1)=v(t) \Delta+x(t) \\
v(t+1)=-\Delta \frac{k_{1} k_{2} \psi_{f}^{2}}{R m} v(t)+v(t)+\Delta \frac{k_{2} \psi_{f}}{R m} u(t) \\
y(t)=v(t)
\end{array}\right.
$$

where $\tau=0.031 \mathrm{~m}, R=8.6 \Omega, m=1.635 \mathrm{~kg}$ and $\psi_{f}=0.35 \mathrm{~Wb}$, respectively. It is easy to find that (37) can be described as a kind of system as (1) with $A=\left[\begin{array}{cc}1 & \Delta \\ 0 & 1-\Delta \frac{k_{1} k_{2} \psi_{f}^{2}}{R m}\end{array}\right], B=\left[\begin{array}{c}0 \\ \Delta \frac{k_{2} \psi_{f}}{R m}\end{array}\right]$ and $C=$ $\left[\begin{array}{ll}0 & 1\end{array}\right]$. From (37), we can see that the product of the output/input coupling matrix $C B=0.0378$ is full column rank.

The iteration-varying trajectory is given by

$$
y_{k+1}^{r}(t)=2 \cos (10 \Delta) y_{k}^{r}(t)-y_{k-1}^{r}(t) .
$$

And the desired trajectories of the first iteration and second iteration are

$$
\begin{aligned}
& y_{1}^{r}(t)=-0.2 \Delta^{2}\left(60 \Delta t^{3}-30 \Delta^{2} t^{4}-30 t^{2}\right) \\
& y_{2}^{r}(t)=-0.2 \Delta^{2}\left(60 \Delta t^{3}-31 \Delta^{2} t^{4}-28 t^{2}\right) .
\end{aligned}
$$

The HOIM-based iterative learning control is designed as

$$
\begin{aligned}
u_{k+1}(t)= & 2 \cos (10 \Delta) u_{k}(t)-u_{k-1}(t)+ \\
& 35 e_{k}(t+1)-28 e_{k-1}(t+1)
\end{aligned}
$$

We choose the learning control gain as $\gamma_{1}=35$ and $\gamma_{2}=-28$ so that $\left\|2 \cos (10 \Delta)-C B \gamma_{1}\right\|=0.667<1$ and $\left\|-1-C B \gamma_{2}\right\|=0.058<1$. The corresponding characteristic polynomial is $z^{2}-0.667 z-0.058$ with two eigenvalues inside the unit circle.

Fig. 1 shows that the desired trajectories are changing in the iteration domain continuously. Let $e_{k, \max }=$ $\max _{t \in[0, T]}\left|e_{k}(t)\right|$ be maximum absolute tracking error of time interval $t \in[0, T]$ of the $k$-th iteration. The tracking results of HOIM-based ILC are illustrated in Fig. 2. The effectiveness of HOIM can be clearly seen.

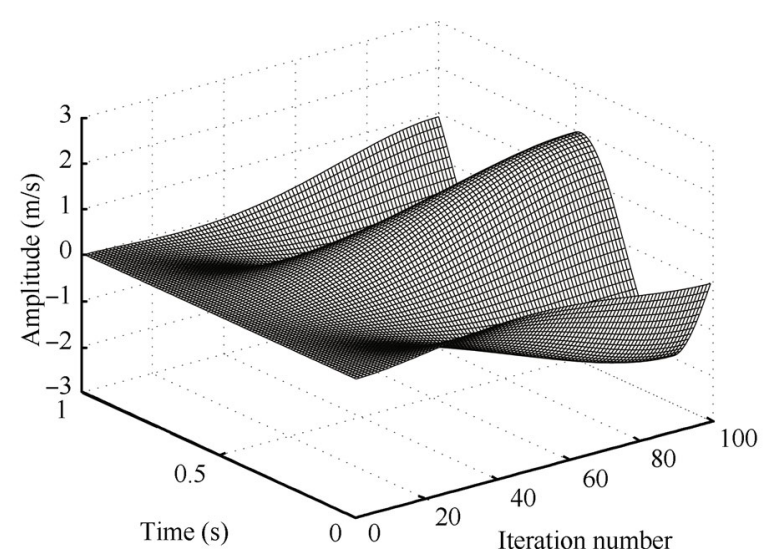

Fig. 1 Iteratively varying reference trajectory

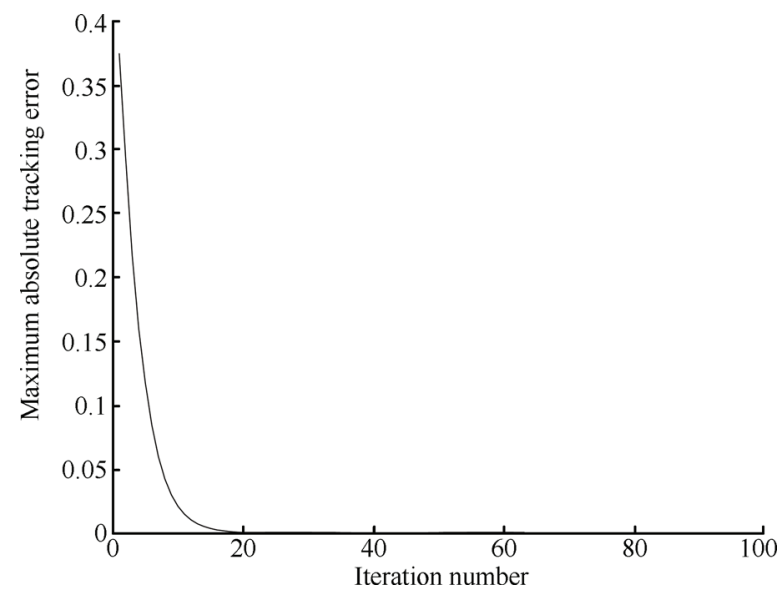

Fig. 2 The maximum absolute tracking error along the iteration axis

To show the time-domain behavior, Fig. 3 gives the tracking profiles at the 4 th, 7 th and 20 th iterations. It can be seen from Fig. 3 that the 7 th tracking profile is close to the reference trajectory and the 20th output curve coincides with tracking trajectory. The HOIM based control scheme shows the perfect tracking performance for the discretized PMLM systems (32) to track iteration-varying desired trajectories shown in Fig. 1.

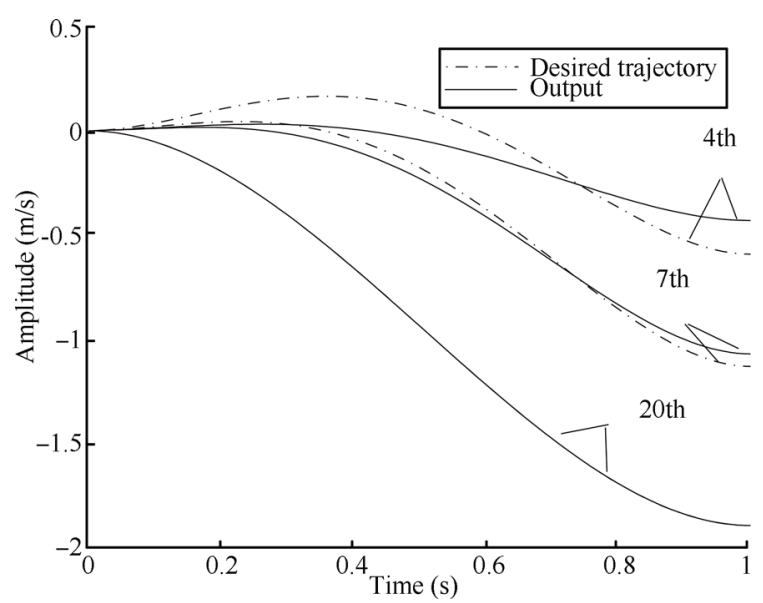

Fig. 3 Tracking profiles of the HOIM-based ILC for the 4th, 7th and 20th iterations

\section{Conclusions}

An HOIM-based ILC scheme is proposed for a class of discrete-time linear time-varying (LTV) systems with relative degree of one to track iteration-varying desired trajectories. It is shown that under some sufficient conditions on the learning operators, the convergence of the learning system can be guaranteed. The proposed ILC algorithm is applied to the tracking control of PMLM. Simulation results confirm the efficacy of the proposed ILC method. 


\section{References}

[1] M. Uchiyama. Formation of high speed motion pattern of mechanical arm by trial. Transactions of the Society of Instrumentation and Control Engineers, vol. 14, no. 6, pp. 706-712, 1978. (in Japanese)

[2] S. Arimoto, T. Naniwa, H. Suzuki. Robustness of P-type learning control with a forgetting factor for robotic motions. In Proceedings of the 29th Conference on Decision and Control, IEEE, Honolulu, USA, pp. 2640-2645, 1990.

[3] K. L. Moore, M. Dahleh, S. P. Bhattacharyya. Iterative learning control: A survey and new results. Journal of Robotic Systems, vol. 9, no. 5, pp. 563-594, 1992.

[4] M. Yu, J. S. Wang, D. L. Qi. Output-feedback adaptive learning control with unknown control direction. Journal of Zhejiang University (Engineering Science), vol. 47, no. 8, pp. 1424-1430, 2013. (in Chinese)

[5] D. Q. Huang, X. F. Li, J. X. Xu, C. Xu, W. He. Iterative learning control of inhomogeneous distributed parameter systems-frequency domain design and analysis. Systems \& Control Letters, vol. 72, pp. 22-29, 2014.

[6] S. Arimoto. Robustness of learning control for robot manipulators. In Proceedings of IEEE International Conference on Robotics and Automation, IEEE, Cincinnati, USA, pp. 1528-1533, 1990.

[7] X. D. Li, T. F. Xiao, H. X. Zheng. Adaptive discrete-time iterative learning control for non-linear multiple input multiple output systems with iteration-varying initial error and reference trajectory. IET Control Theory \& Applications, vol. 5, no. 9, pp. 1131-1139, 2011.

[8] Z. S. Hou, J. X. Xu, H. W. Zhong. Freeway traffic control using iterative learning control-based ramp metering and speed signaling. IEEE Transactions on Vehicular Technology, vol. 56, no. 2, pp. 466-477, 2007.

[9] D. Q. Huang, J. X. Xu. Steady-state iterative learning control for a class of nonlinear PDE processes. Journal of Process Control, vol. 21, no. 8, pp. 1155-1163, 2011.

[10] D. Q. Huang, J. X. Xu, V. Venkataramanan, T. C. T. Huynh. High-performance tracking of piezoelectric positioning stage using current-cycle iterative learning control with gain scheduling. IEEE Transactions on Industrial Electronics, vol. 61, no. 2, pp. 1085-1098, 2014.

[11] D. Q. Huang, J. X. Xu, X. F. Li, C. Xu, M. Yu. D-type anticipatory iterative learning control for a class of inhomogeneous heat equations. Automatica, vol. 49, no. 8, pp. 2397$2408,2013$.

[12] S. Arimoto. Learning control theory for robotic motion. International Journal of Adaptive Control and Signal Processing, vol. 4, no. 6, pp. 543-564, 1990.

[13] Y. Q. Chen, C. Y. Wen, M. X. Sun. A robust high-order P-type iterative learning controller using current iteration tracking error. International Journal of Control, vol. 68, no. 2, pp. 331-342, 1997.

[14] Y. Q. Chen, Z. M. Gong, C. Y. Wen. Analysis of a highorder iterative learning control algorithm for uncertain nonlinear systems with state delays. Automatica, vol. 34, no. 3, pp. 345-353, 1998.

[15] X. H. Bu, Z. S. Hou. Stability of iterative learning control with data dropouts via asynchronous dynamical system. International Journal of Automation and Computing, vol. 8, no. 1, pp. 29-36, 2011.
[16] D. Q. Huang, Y. Tan, J. X. Xu. A dual-loop iterative learning control for nonlinear systems with hysteresis input uncertainty. In Proceedings of IEEE International Conference on Control and Automation, IEEE, Christchurch, New Zealand, pp. 1116-1121, 2009.

[17] K. L. Moore, M. H. A. Verwoerd. $L_{1}$-optimal robust iterative learning controller design. In Proceedings of the American Control Conference, IEEE, Seattle, USA, pp. 38813886, 2008.

[18] W. S. Chen. Novel adaptive learning control of linear systems with completely unknown time delays. International Journal of Automation and Computing, vol. 6, no. 2, pp. 177-185, 2009.

[19] W. S. Chen, R. H. Li, J. Li. Observer-based adaptive iterative learning control for nonlinear systems with timevarying delays. International Journal of Automation and Computing, vol. 7, no. 4, pp. 438-446, 2010.

[20] J. M. Li, X. M. Li, K. Xing. Hybrid adaptive iterative learning control of non-uniform trajectory tracking for nonlinear time-delay systems. In Proceedings of the 26th Chinese Control Conference, IEEE, Zhangjiajie, China, pp. 515-519, 2007.

[21] M. Yu, J. L. Zhang, D. L. Qi. Discrete-time adaptive iterative learning control with unknown control directions. International Journal of Control, Automation and Systems, vol. 10, no. 6, pp. 1111-1118, 2012.

[22] R. H. Chi, D. W. Wang, Z. S. Hou, S. T. Jin, D. X. Zhang. A discrete-time adaptive iterative learning from different reference trajectory for linear time-varying systems. In Proceedings of the 31st Chinese Control Conference, IEEE, Hefei, China, pp. 3013-3016, 2012.

[23] Y. Q. Chen, K. L. Moore. Harnessing the non-repetitiveness in iterative learning control. In Proceedings of the 41st IEEE Conference on Decision and Control, IEEE, Las Vegas, USA, pp. 3350-3355, 2002.

[24] K. L. Moore, Y. Q. Chen, H. S. Ahn. Iterative learning control: A tutorial and big picture view. In Proceedings of the 45th IEEE Conference on Decision and Control, IEEE, San Diego, USA, pp. 2352-2357, 2006.

[25] A. Tayebi, M. B. Zaremba. Internal model-based robust iterative learning control for uncertain LTI systems. In Proceedings of the 39th IEEE Conference on Decision and Control, IEEE, Sydney, Australia, pp. 3439-3444, 2000.

[26] W. Zhou, M. Yu, D. L. Qi. Iterative learning control design with high-order internal model for permanent magnet linear motor. In Proceedings of International Conference on Life System Modeling and Simulation and International Conference on Intelligent Computing for Sustainable Energy and Environment, Springer-Verlag, Shanghai, China, pp. 208-217, 2014.

[27] C. P. Liu, J. X. Xu, J. Wu. On iterative learning control with high-order internal models. International Journal of Adaptive Control and Signal Processing, vol. 24, no. 9, pp. 731-742, 2010.

[28] C. K. Yin, J. X. Xu, Z. S. Hou. On iterative learning control design for tracking iteration-varying trajectories with high-order internal model. Journal of Control Theory and Applications, vol. 8, no. 3, pp. 309-316, 2010.

[29] C. K. Yin, J. X. Xu, Z. S. Hou. A high-order internal model based iterative learning control scheme for nonlinear systems with time-iteration-varying parameters. IEEE Transactions on Automatic Control, vol.55, no.11, pp. 26652670, 2010. 
[30] Y. Fang, T. W. S. Chow. Iterative learning control of linear discrete-time multivariable system. Automatica, vol. 34, no. 11, pp. 1459-1462, 1998.

[31] M. X. Sun, D. W. Wang. Initial shift issues on discrete-time iterative learning control with system relative degree. IEEE Transactions on Automatic Control, vol. 48, no. 1, pp. 144148, 2003.

[32] C. J. Chien. A discrete iterative learning control for a class of nonlinear time-varying systems. IEEE Transactions on Automatic Control, vol. 43, no. 5, pp. 748-752, 1998.

[33] D. W. Wang. Convergence and robustness of discrete time nonlinear systems with iterative learning control. Automatica, vol. 34, no. 11, pp. 1445-1448, 1998.

[34] R. H. Chi, Z. S. Hou, J. X. Xu. Adaptive ILC for a class of discrete-time systems with iteration-varying trajectory and random initial condition. Automatica, vol.44, no. 8, pp. 2207-2213, 2008.

[35] M. Yu, J. S. Wang, D. L. Qi. Discrete-time adaptive iterative learning control for high-order nonlinear systems with unknown control directions. International Journal of Control, vol. 86, no. 2, pp. 299-308, 2013.

[36] M. Yu, J. S. Wang, D. L. Qi. Feedback nonlinear discretetime systems. International Journal of Systems Science, vol. 45, no. 11, pp. 2251-2259, 2014.

[37] K. L. Moore. A matrix fraction approach to higher-order iterative learning control: 2-D dynamics through repetitiondomain filtering. In Proceedings of the 2nd International Workshop on Multidimensional Systems, IEEE, Czocha Castle, Poland, pp. 99-104, 2000.

[38] S. S. Saab. A discrete-time learning control algorithm for a class of linear time-invariant systems. IEEE Transactions on Automatic Control, vol. 40, no. 6, pp. 1138-1142, 1995.

[39] S. T. Jin, Z. S. Hou, R. H. Chi, Y. Q. Li. Discrete-time adaptive iterative learning control for permanent magnet linear motor. In Proceedings of the 5th IEEE International Conference on Cybernetics and Intelligent Systems, IEEE, Qingdao, China, pp. 69-74, 2011.

[40] K. K. Tan, S. Y. Lim, S. N. Huang. Two-degree-of-freedom controller incorporating RBF adaptation for precision motion control applications. In Proceedings of IEEE/ASME International Conference on Advanced Intelligent Mechatronics, IEEE, Atlanta, USA, pp. 848-853, 1999.

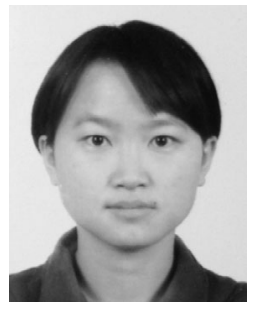

Wei Zhou received B. Sc. degree in power system and automation from North China Electric Power University, China in 1999 , and the M. Sc. degree in power electronics from Nanchang University, China in 2005. From 2006, she is a lecturer in the College of Engineering Jiangsu Institute of Commerce, China. Currently, she is a Ph. D. Candidate in control theory and control engineering in Zhejiang University, China.

Her research interests include adaptive control and iterative learning control.

E-mail: pinkzhouwei@163.com

ORCID iD: 0000-0002-1730-1577

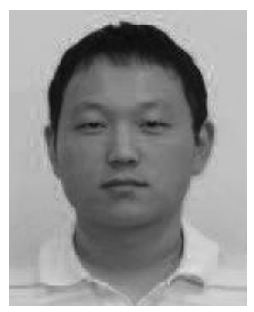

Miao Yu received the B. Sc. and Ph. D degrees in control theory and control engineering from Zhejiang University, China in 2007 and 2012, respectively. Currently he is doing postdoctoral research at Aalto University, Finland.

His research interests include adaptive systems, iterative learning control and robot manipulators.

E-mail: zjuyumiao@gmail.com (Corresponding author) ORCID iD: 0000-0002-7638-5264

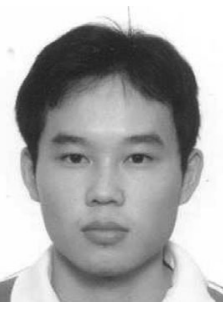

De-Qing Huang received the Bachelor and the first Ph. D. degrees from the Mathematical College of Sichuan University, China in 2002 and 2007, respectively. He attended the Department of Electrical and Computer Engineering, National University of Singapore, Singapore in 2006, where he received the second $\mathrm{Ph}$. D. degree in control engineering in 2011. Now, he is a research associate in the Department of Aeronautics, Imperial College London, UK.

His research interests include dynamic analysis and bifurcation, fluid flow analysis and control, learning theory, nonlinear and robust control, applications to servo and drives, batch reactors, and industrial processes.

E-mail: elehd2012@gmail.com

ORCID iD: 0000-0002-8185-9030 